\title{
Differential Geometry and Acoustics: A Survey
}

\author{
David R. Bergman \\ Exact Solution Scientific Consulting LLC, 37 Davenport Place, Morristown NJ 07960, USA. \\ E-mail: davidrbergman@essc-llc.com.
}

(Received 28 January 2020; accepted 20 February 2021)

A connection between acoustic rays in a moving inhomogeneous fluid medium and the null geodesic of a pseudoRiemannian manifold provides a mechanism to derive several well-known results commonly used in acoustic ray theory. Among these include ray integrals for depth dependent sound speed and current profiles commonly used in ocean and aero acoustic modelling. In this new paradigm these are derived by application of a symmetry of the effective metric tensor known as isometry. In addition to deriving well-known results, the application of the full machinery of differential geometry offers a unified approach to modelling acoustic fields in three dimensional random environments with time dependence by, (1) using conformal symmetry to simplify the geodesic equation, and (2) application of geodesic deviation as a generalization of geometric spread.

\section{INTRODUCTION}

The connection between differential geometry and ray theory can be traced to the method of characteristics applied to a hyperbolic partial differential equation (PDE). ${ }^{1,2}$ The equations of fluid mechanics and electrodynamics are of this type ${ }^{1-3}$ For a field depending on three coordinates and time, the characteristics of the PDE are surfaces in 3D space generated by advancing the surface of initial data forward into the medium for future values of time. The bicharacteristics are a family of curves that define the push forward map for the initial surface. Ray theory emerges as a high frequency approximation of the wave equation by assuming an infinite series in powers of $\omega^{-1}$, where $\omega$ is the angular frequency. The coefficients of this series satisfy a hierarchy of equations with the lowest order term, the eikonal, defining the ray path geometry. The next term in the series accounts for the lowest order amplitude, related to the geometric spread of a bundle of ray paths with similar initial conditions. The eikonal equation has the same form as the characteristic equation, and it is through this equation that the concept of an acoustic metric emerges.

Specific applications of differential geometry in acoustics appear in a variety of fields. The identification of ray paths with geodesics of an abstract metric space has been presented in acoustics by several authors. In a 1972 paper by R. White, this approach is used to develop a 4D ray tracing procedure for acoustic rays in a moving fluid which makes use of a Lorentzian (also called a pseudo-Riemannian) manifold. ${ }^{4}$ Babich and Kiselev identify rays in elastic vibrations with geodesics of a Riemannian manifold. ${ }^{5}$ Meyer and Schroeter produced a similar result as White in their 1986 paper for ray tracing in a moving fluid medium. ${ }^{6}$ In this case, the authors identified the ray paths with geodesics of a Finsler space. In the field of general relativity, a seminal paper published by Unruh in 1982 makes the same connection between acoustics and differential geometry for the purpose of developing fluid mechanical models of fields propagating near black hole event horizons. ${ }^{7}$ Recently, the use of Lorentzian manifolds has led to the development of a 4D paraxial ray tracing algorithm. ${ }^{8,9}$

This paper provides a review of research on differential geometry (DG) and its application to acoustics. An introduction to this topic was presented at ICSV 22; this paper contains both a deeper and broader presentation of the material. ${ }^{10}$ The intent is to impress upon the reader that DG is a useful conceptual tool for dealing with problems in wave propagation in an inhomogeneous moving fluid medium. Due to space constraints, the presentation will not include complete derivations of the results and the reader is referred to the literature for details ${ }^{4-13}$. What the reader will find here is an organized list of known results and articles relevant to the topic, along with a short description of applications. The paper is divided into sections as follows. Section 2 discusses the type of geometry induced by the equations of fluid dynamics. Section 3 presents several known results derived from the acoustic metric. Section 4 discusses conformal symmetry in ray theory. In Section 5 ., the concept of 4D paraxial or dynamic ray tracing is introduced. Section 6. discusses the relationship between, (1) DG and chaos theory, and (2) Gaussian beam tracing and geodesic deviation.

\section{ACOUSTIC GEOMETRY}

\subsection{Geometry and Wave Propagation}

It is important to distinguish between the different uses of DG in acoustics since it appears in more than one context. Common to all theories of wave propagation are the ideas of wavefronts and ray paths. ${ }^{14}$ One is accustomed to describing the propagation of wavefronts from a source as $2 \mathrm{D}$ surfaces immersed in a $3 \mathrm{D}$ space. As time evolves, a wavefront will advance through the medium, changing shape along the way as it encounters boundaries, changes in refractive index and fluid flow, and any other physical interactions that affect propagation. At any instant the wavefront can be described using the theory of surfaces, providing a complete description of the geometry of the wavefront as a parameterized surface in $3 \mathrm{D}, f(u, v)$, or a constrained position vector, $\vec{r}(u, v)$, where $u$ and $v$ are two independent coordinates on the surface. A frame consisting of the unit normal vector to the wavefront, $\hat{n}=\vec{\nabla} f /\|\vec{\nabla} f\|$, and a set of unit tangent vectors, $\hat{e}_{k}=\partial_{k} \vec{r} /\left\|\partial_{k} \vec{r}\right\|(k \in\{u, v\})$ can be constructed at every point on the wavefront except at a caustic. The geometry of these surfaces may be curved but the space they move 


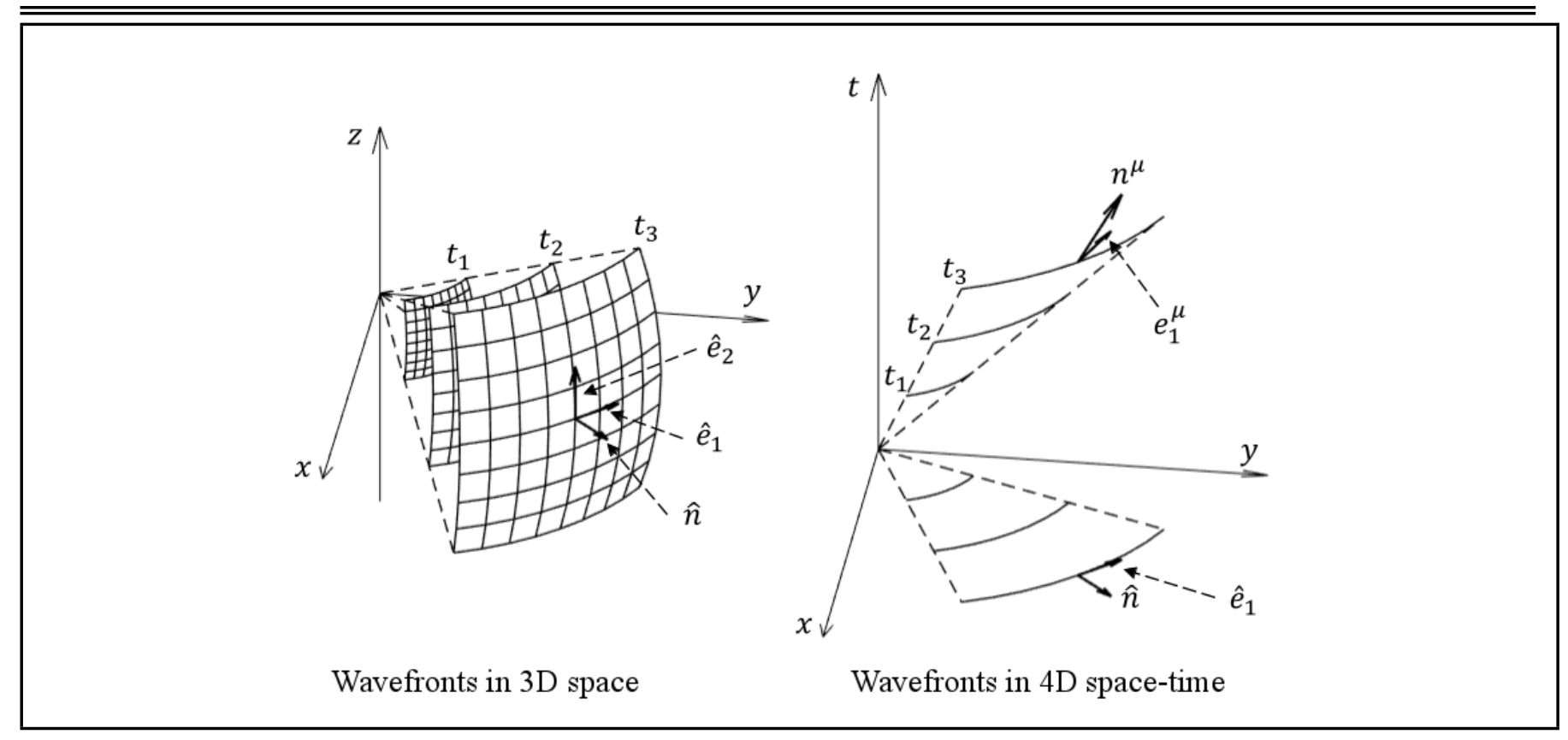

Figure 1. Example of wavefronts in 3D space (left) and 4D space-time (right).

through is flat Euclidean space. Using the parameterized vector $\vec{r}(u, v)$ one can develop a description of the surface geometry, including a metric tensor $g_{\mathrm{u}}=\partial_{u} \vec{r} \cdot \partial_{v} \vec{r}$, Christoffel symbols, and curvature. ${ }^{15,16}$ Similarly, the geometry of the ray paths as curves in 3D Euclidean space is described by the Frenet-Serret formulas. ${ }^{15}$

The generalization of the theory of surfaces to abstract manifolds that are locally Euclidian is known as Riemannian geometry. In contrast, the type of geometry that arises naturally in the study of hyperbolic partial differential equations does not fall under this category. Rather, one has a description that treats space and time as parameters on equal footing and descriptions of hyper-surfaces immersed in 4D space-time.

These two descriptions are illustrated in Figure 1. A propagating wavefront is shown on the left at three times $\left\{t_{1}, t_{2}, t_{3}\right\}$, along with the frame field at the center point of the surface at $t_{3}$. The corresponding 4D space-time description is shown on the right for the same set of wavefronts (with the $z$-axis suppressed). As a matter of convention, time and space taken separately are labelled $(t, \vec{x})$, whereas space-time coordinates are denoted $x^{\mu}$, with $\mu=0-3$, where $\mu=0$ is reserved for time and indices 1-3 indicate spatial coordinates. An exceptional presentation of ray and wavefront geometry in Euclidian 3D space can be found in Stavroudis. ${ }^{17}$ The remainder of this review is concerned with the latter type of geometric abstraction.

\subsection{The Method of Characteristics and Acoustics}

Consider an inviscid, isentropic, Newtonian fluid complying with the equation of continuity and Euler's equation of motion. The variables describing the fluid are density $\rho$, pressure $p$, and particle velocity $\vec{v}$. The isentropic assumption implies an equation of state in which pressure $p$ depends only on density $\rho$, leading to the definition of sound speed as a function of position and time; $c^{2}=d p / d \rho$. Under these assumptions, the fluid complies with Eqs. (1) and (2).

$$
\begin{gathered}
\frac{\partial p}{\partial t}+\vec{v} \cdot \vec{\nabla} p+\rho c^{2} \vec{\nabla} \cdot \vec{v}=0 ; \\
\rho\left(\frac{\partial \vec{v}}{\partial t}+\vec{v} \cdot \vec{\nabla} \vec{v}\right)+\vec{\nabla} p=-\vec{\nabla} \Phi .
\end{gathered}
$$

In Eq. (2), the term on the r.h.s. represents external conservative forces, such as gravity, acting on the fluid. The method of characteristics is applied to Eqs. (1) and (2) by constructing a vector $u_{n}$ from the variables $u_{n}=(p, \vec{v}), u_{1}=p, u_{2}=v_{x}$, $u_{3}=v_{y}$, and $u_{4}=v_{z}$. each a function of $x^{\mu}$, leading to a first order PDE system of the form $A_{\mathrm{mn}}^{\nu} \partial_{\nu} u_{n}+b_{m}=0$ (the Einstein summation convention is used for both Greek and Latin indices). The terms $A_{\mathrm{nm}}^{\nu}$ and $b_{m}$ are functions of $x^{\nu}$ and $u_{n}$ but not the derivatives of $u_{n}$. For details on the method of characteristics the reader is referred to Courant and Hilbert, Volume II. ${ }^{1}$ A hyper-surface, $\varphi\left(x^{\nu}\right)$, is defined and the characteristic determinant $A=\operatorname{det}\left(A^{\nu} \partial_{\nu} \varphi\right)$ is formed. Setting the determinant equal to zero defines the characteristic equation, a first order non-linear PDE satisfied by $\varphi\left(x^{\nu}\right)$, referred to as a characteristic surface. When applied to Eqs. (1) and (2) the result is $(\mathrm{D} \phi)^{2}\left((\mathrm{D} \phi)^{2}-c^{2} \vec{\nabla} \varphi \cdot \vec{\nabla} \varphi\right)=0$, where the material derivative operator $D$ is defined by $D \varphi=$ $\frac{\partial \varphi}{\partial t}+\vec{v} \cdot \vec{\nabla} \varphi$. A partial Hamiltonian is defined for the second factor $H=-\left((\mathrm{D} \phi)^{2}-c^{2} \vec{\nabla} \varphi \cdot \vec{\nabla} \varphi\right)$, which may be expressed compactly as $H=g^{\mu \nu} \partial_{\mu} \varphi \partial_{\nu} \varphi=0\left(\partial_{\mu}=\frac{\partial}{\partial x^{\mu}}\right)$ formally introducing the contravariant metric, $g^{\mu \nu}$, the sign chosen to maintain consistency with the sign convention in the definition of the acoustic metric (to be defined later in this paper). The bicharacteristics are a set of parameterized curves in space-time, $x^{\mu}(\lambda)$, parameterized by an arbitrary parameter $\lambda$, not to be confused with wavelength. The 4D gradient of the characteristic is identified with a momentum variable conjugate to $\frac{d x^{\mu}}{d \lambda}$ via $p_{\nu}=\partial_{\nu} \varphi$. The equations of motions for these variables are derived using the general theory of Hamiltonian 
mechanics.

$$
\begin{gathered}
\frac{d x^{\mu}}{d \lambda}=\frac{1}{2} \frac{\partial H}{\partial p_{\mu}}=g^{\mu \nu} p_{\nu} \\
\frac{d p_{\mu}}{d \lambda}=-\frac{1}{2} \frac{\partial H}{\partial x^{\mu}}=-\frac{1}{2} p_{\beta} p_{\alpha} \frac{\partial g^{\alpha \beta}}{\partial x^{\mu}} .
\end{gathered}
$$

Equation (3) relates the generalized momentum to the velocity and can be inverted using the relation $g^{\mu \alpha} g_{\alpha \nu}=\delta_{\nu}^{\mu}$ to give $p_{\mu}=g_{\mu \nu} \frac{d x^{\nu}}{d \lambda}$ and used to convert Eq. (4) to a second order ordinary differential equation for the curve $x^{\mu}(\lambda)$. The process requires some algebra and the introduction of more derivatives of the metric tensor with respect to coordinates. The result is the geodesic equation.

$$
\frac{d^{2} x^{\mu}}{d \lambda^{2}}+\Gamma_{\alpha \beta}^{\mu} \frac{d x^{\alpha}}{d \lambda} \frac{d x^{\beta}}{d \lambda}=0
$$

In Eq. (5) $2 \Gamma^{\mu}{ }_{\alpha \beta}=g^{\mu \nu}\left(\partial_{\alpha} g_{\nu \beta}+\partial_{\beta} g_{\nu \alpha}-\partial_{\nu} g_{\alpha \beta}\right)$ is the Christoffel symbol of the second kind. Referring back to the form of $H$ one can derive the following expressions in matrix form for the contravariant and covariant metric tensors.

$$
g_{\mu \nu}=\left(\begin{array}{cc}
-\left(c^{2}-v^{2}\right) & -\vec{v}^{T} \\
-\vec{v} & \mathbf{I}
\end{array}\right) ; g^{\mu \nu}=\frac{1}{c^{2}}\left(\begin{array}{cc}
-1 & -\vec{v}^{T} \\
-\vec{v} & \mathbf{I} c^{2}-\vec{v} \vec{v}^{T}
\end{array}\right)
$$

In Eq. (6) I represents a 3D identity matrix, also indicated by some authors by the Kronecker Delta $\delta_{\mathrm{ij}}$. As a final point, writing the original characteristic equation in terms of $\frac{d x^{\nu}}{d \lambda}$ rather than $\partial_{\nu} \varphi$ leads to the following constraint obeyed by the bicharacteristic curves. $1,4,7-9$

$$
\begin{aligned}
\mathrm{dS}^{2}= & g_{\mu \nu} d x^{\mu} d x^{\nu}= \\
& -\left(c^{2}-v^{2}\right) \mathrm{dt}^{2}-2(\vec{v} \cdot d \vec{x}) d t+d \vec{x} \cdot d \vec{x}=0 .
\end{aligned}
$$

The symbol dS refers to a differential element of arclength in $4 \mathrm{D}$, which is a generalization of the usual definition of arclength in 3D given by $d s^{2}=d \vec{x} \cdot d \vec{x}$. The dot product appearing in the second and third terms of Eq. (7) is the ordinary Euclidian norm. In the remainder of the paper, the flow is restricted to be subsonic but not necessarily low velocity. With this type of metric, the norm of a vector is no longer positive definite, and the bicharacteristics of the hyperbolic PDE are restricted to have zero length in 4D. In Eq. (5) a generic independent parameter $\lambda$, called an affine parameter, is introduced, which belongs to an equivalence class defined by $\{a \lambda+b\}$ with $a$ and $b$ real numbers. ${ }^{15,18}$ Together, Eq. (5) and Eq. (7) state that the bicharacteristics of Eqs. (1) and (2) are null geodesics of a manifold with metric given by Eq. (6).

\subsection{The Wave Equation}

In the last section no assumptions were made regarding the size of the acoustic field. It is common in practice to consider the field variables as consisting of background values, $\left\{\rho_{0}, p_{0}, \vec{v}_{0}\right\}$, and small perturbations $\left\{\rho_{1}, p_{1}, \vec{v}_{1}\right\}$, e.g., $p=p_{0}+p_{1}$ etc. The equation of state allows $\rho_{1}$ to be eliminated as a degree of freedom via the relation $p_{1}=c^{2} \rho_{1}$. Background values are assumed to obey Eqs. (1) and (2) independently of the perturbations, and the perturbations, assumed small enough to neglect nonlinear terms, are identified with linear acoustics. These assumptions lead to the following equations describing acoustic propagation in a generic moving fluid medium (external forces are omitted in this discussion).

$$
\begin{array}{r}
\left(D_{0} p_{1}+\rho_{0} c^{2} \vec{\nabla} \cdot \vec{v}_{1}\right) \\
+\left(\vec{v}_{1} \cdot \vec{\nabla} p_{0}+p_{1} \vec{\nabla} \cdot \vec{v}_{0}+p_{1} c^{2} D_{0} c^{-2}\right)=0 \\
\left(D_{0} \vec{v}_{1}+\frac{1}{\rho_{0}} \vec{\nabla} p_{1}\right)+\left(\vec{v}_{1} \cdot \vec{\nabla} \vec{v}_{0}+\frac{p_{1}}{\rho_{0} c^{2}} D_{0} \vec{v}_{0}\right)=0
\end{array}
$$

The material derivative with respect to the background flow is defined by $D_{0} f=\frac{\partial f}{\partial t}+\vec{v}_{0} \cdot \vec{\nabla} f$. Application of the method of characteristics to Eqs. (8) and (9) leads to the same result for the geometry of the bicharacteristics with all fields in the metric tensor replaced with background values. By differentiating Eqs. (8) and (9) second order equations can be derived for the acoustics perturbations. This approach is taken by Unruh with the additional assumption of irrotational flow, $\vec{\nabla} \times \vec{v}=0$, applied prior to perturbing the fields and perturbations applied to the variables $\psi(\vec{v}=\vec{\nabla} \psi)$ and $\xi=\ln \rho$, with $p(\rho)$, to derive the following wave equation for $\psi_{1}$.

$$
\frac{1}{\sqrt{|g|}} \partial_{\mu} \sqrt{|g|} g^{\mu \nu} \partial_{\nu} \psi_{1}=0
$$

The main result is that the full wave equation is converted into a covariant differential equation with the operator $|g|^{-1 / 2} \partial_{\mu} \sqrt{|g|} g^{\mu \nu} \partial_{\nu}$ defined on a 4D space-time manifold. The factor $|g|$ is the determinant of the covariant metric tensor, which in this case takes a different form than Eq. (6).

$$
g_{\mu \nu}=\frac{\rho_{0}}{c}\left(\begin{array}{cc}
\left(c^{2}-v_{0}^{2}\right) & \vec{v}_{0}^{T} \\
\vec{v}_{0} & -\mathbf{I}
\end{array}\right)
$$

For a complete derivation, the reader is referred to Unruh's paper, the main result being presented here for discussion in later sections. ${ }^{7}$ Before discussing ray theory, mention is made of the fact that the background fluid density appears in Eq. (11) but not in Eq. (6). This will be discussed again later but it is noted that the bicharacteristics defined as curves of zero length, obeying the constraint Eq. (7), are unaffected by an overall multiplicative factor. The full wave equation derived by Unruh, Eq. (10), does not share this invariance and is dependent on background fluid density and its derivative.

\subsection{Ray Theory}

Ray theory remerges from Eqs. (8) and (9) by assuming a solution for the fields in the form of a one parameter family of approximate-plane-waves to the fields, $p_{1}=\pi_{\alpha} e^{i \varphi / \alpha}$ and $\vec{v}_{1}=\vec{\sigma}_{\alpha} e^{i \varphi / \alpha}$, with $\pi_{\alpha}=\pi_{0}+\alpha \pi_{1}+\cdots, \vec{\sigma}_{\alpha}=\vec{\sigma}_{0}+\alpha \vec{\sigma}_{1}+\cdots$, and taking the limit of small $\alpha$. A detailed treatment of the approach may be found in Perlick. ${ }^{19}$ Prior to this, Eqs. (8) and (9) are converted to second order equations for the field $p_{1}$ and the velocity $\vec{v}_{1}$. A detailed derivation of the linear equations for acoustic and the eikonal approximation applied to them can be found in appendices B.2 and B.3 of reference [8]. Results are presented here to motivate discussion.

$$
D_{0}\left(\frac{1}{\rho_{0} c^{2}} D_{0} p_{1}\right)-\vec{\nabla} \cdot\left(\frac{1}{\rho_{0}} \vec{\nabla} p_{1}\right)+F=0
$$




$$
D_{0}\left(\rho_{0} D_{0} \vec{v}_{1}\right)-\vec{\nabla}\left(\rho_{0} c^{2} \vec{\nabla} \cdot \vec{v}_{1}\right)+\vec{F}=0 .
$$

The terms $F$ and $\vec{F}$ contain first derivatives of $p_{1}$ and $\vec{v}_{1}$ as well as the background fields and their derivatives, but the explicit forms are not relevant to the discussion. The lowest order term in the expansion leads to the eikonal equation $g^{\mu \nu} \partial_{\mu} \varphi \partial_{\nu} \varphi=0$ with the background fields appearing in the acoustic metric, Eq. (6). The solution to the eikonal equation is again described by the same geometry as the characteristics with the effective metric containing $\vec{v}_{0}\left(x^{\mu}\right)$ and $c\left(x^{\mu}\right)$. In addition to a geometric description of ray paths, a first order approximation of the amplitude is obtained from the rate of geometric spread of the ray paths and the Blokhintzev invariant. ${ }^{20}$ These two terms are the foundation of ray theoretic estimates of acoustic pressure except near caustics where higher order corrections are required. ${ }^{21}$

In 1971, the geometric description presented in sections 2.2 . and 2.4. was introduced by R. W. White in a novel approach to ray tracing. ${ }^{4}$ Virtues of the approach are that it does not include the assumptions of weak flow and the approach holds for the full non-linear theory. Therefore, the approach represents the most general description of ray paths under the assumptions made in section 2.2. In 1981, Meyer and Schroeter developed a ray trace procedure which identified the rays in a moving fluid as geodesics of a Finsler space. ${ }^{6}$ Finsler and Lorentzian manifolds are both extensions of Riemannian manifolds, each with distinct novel characteristics. Lorentzian manifolds introduce the concept of zero length vectors and null hyper-spaces, while Finsler manifolds introduce the need for a metric that depends locally on the geodesic velocity. A point that cannot be over stressed is that rays and bicharacteristics are geodesics of a Lorentzian manifold even when $\vec{\nabla} \times \vec{v} \neq 0$. In contrast, the full wave equation presented in Eq. (10) is defined on a differential manifold when $\vec{\nabla} \times \vec{v}=0$. It is this feature that makes Eq. (10) of value as a toy model in general relativity and quantum field theory. Rays will always behave like geodesics on a Lorentzian manifold whereas acoustic waves live on a Lorentzian manifold only for irrotational flow. A second point is that the metric appearing in Eq. (11) contains the background density, whereas that in Eq. (6) does not. The full wave equation couples to the density gradients as expressed in the original equations.

It is worth comparing the approach presented here to other authors. An article by Blokhintzev provides a rigorous treatment of acoustics starting from the equation of continuity, Euler's equation, and an equation of state that includes entropy. ${ }^{20}$ This treatment is more general in that the linearization of the equations, as well as the eikonal approximation, include entropy. The zeroth order solution turns out to be isentropic, emerging as a solution rather than an assumption. The treatment appearing in White ${ }^{4}$ is valid for the full non-linear theory, a result not considered in previous work. A feature of both the method of characteristics and the development of ray theory in the eikonal approximation is that only the highest order derivatives in the original equations contribute to the final form of the equation for the bicharacteristics or rays. As a result, the specific form of the equation of state would not affect the geometry of the rays or bicharacteristics. In any case, the rays and bicharacteristics are curves of zero length in space-time and invariant under coordinate dependent scale changes. This is known in differential geometry as conformal symmetry. ${ }^{12}$
Regardless of the form of the second order wave equation the ray paths will always have this symmetry.

\section{THE METRIC AS A CONCEPTUAL TOOL}

Well-known results from the study of layered media and their generalizations can be derived from the acoustic metric and theorems from DG..$^{7-9,11}$ The propagation time along a ray path follows immediately from the line element, Eq. (7), by solving for $\mathrm{dt}$,

$$
d t=\frac{ \pm \sqrt{\left(\vec{v} \cdot \frac{d \vec{x}}{d s}\right)^{2}+\left(c^{2}-v^{2}\right)}-\vec{v} \cdot \frac{d \vec{x}}{d s}}{\left(c^{2}-v^{2}\right)} d s ;
$$

where the positive root defines an advancing wavefront. Appealing to the Hamiltonian approach used in the method of characteristics, one can apply Noether's theorem to discover conserved quantities. When a coordinate is absent from the acoustic metric the momentum associated with it is conserved along the bicharacteristic. Missing coordinates are referred to as cyclic, denoted $x^{C}$, and in differential geometry are due to a rigid symmetry of the metric tensor known as an isometry. From Eq. (4) one can see that when $g\left(x^{\mu}\right)$ is independent of a specific coordinate, $x^{C}$, then $\frac{d p_{C}}{d \lambda}=\frac{d\left(g_{\mathrm{C} \beta} \dot{x}^{\beta}\right)}{d \lambda}=0$ (the shorthand $\dot{x}^{\beta}=\frac{d x^{\beta}}{d \lambda}$ is introduced). Even if $g\left(x^{\mu}\right)$ depends on all four coordinates in some representation, there may exist noncoordinate paths along which the metric is invariant. Denoting the tangent vector to such a curve by $\xi_{C}^{\alpha}$, referred to as a Killing vector, the conservation law is expressed relative to this direction as $\frac{d\left(\xi_{C}^{\alpha} g_{\alpha \beta} \dot{x}^{\beta}\right)}{d \lambda}=0$. In general, one can hunt for symmetries by looking for a set of Killing vectors that are solutions to Killing's equation. ${ }^{18}$ Common results from underwater acoustics can be derived using the principle of isometry. ${ }^{21}$ Consider a special case where the environment is described by $\vec{v}=0$ and $c=c(\vec{x})$. Time is a cyclic coordinate and the following constraint follows, $c^{2}\left(\frac{\mathrm{dt}}{d \lambda}\right)=\kappa_{0}$. As an example of the application of isometry consider an environment is described by $c=c(z)$, and $\vec{v}=v_{x}(z) \hat{i}+v_{y}(z) \hat{j}$. In this case, one has $\frac{d\left(g_{0 \beta} \dot{x}^{\beta}\right)}{d \lambda}=\frac{d\left(g_{1 \beta} \dot{x}^{\beta}\right)}{d \lambda}=\frac{d\left(g_{2 \beta} \dot{x}^{\beta}\right)}{d \lambda}=0$. Using these equations and Eq. (7), the depth integrals commonly used for calculating ray paths in a layered media can be derived. In Eqs. (15a) and (15b) the following are defined, $\vec{\alpha}=\frac{\hat{n}_{T}}{(c+\hat{n} \cdot \vec{v})}, \alpha=|\vec{\alpha}|$, with $\hat{n}=\left[\begin{array}{lll}\sin \theta \cos \varphi & \sin \theta \sin \varphi & \cos \theta\end{array}\right]^{T}, \theta, \varphi$ and the polar and azimuth angles in spherical coordinates used here to define the orientation of the vector $\hat{n}$. The unit vector $\hat{n}$ is the wave front normal and $\hat{n}_{T}$ the component of $\hat{n}$ projected into the $x-y$ plane, $\vec{x}_{T}=[x, y]^{T}$. All quantities in the definition of $\vec{\alpha}$ are evaluated at the initial point of the ray and initial angle of the wave front corresponding to the initial launch angle of the ray path, e.g. $\frac{\hat{n}_{T}\left(\theta_{0}, \varphi_{0}\right)}{\left(c\left(z_{0}\right)+\hat{n}\left(\theta_{0}, \varphi_{0}\right) \cdot \vec{v}\left(z_{0}\right)\right)} \cdot{ }^{9}$

$$
\begin{gathered}
\frac{\mathrm{dt}}{\mathrm{dz}}=\frac{(1-\vec{\alpha} \cdot \vec{v})}{c \sqrt{(1-\vec{\alpha} \cdot \vec{v})^{2}-\alpha^{2} c^{2}}} \\
\frac{d \vec{x}_{T}}{\mathrm{dz}}=\frac{\vec{\alpha} c^{2}+\vec{v}(1-\vec{\alpha} \cdot \vec{v})}{c \sqrt{(1-\vec{\alpha} \cdot \vec{v})^{2}-\alpha^{2} c^{2}}}
\end{gathered}
$$

The reader can find similar results for rotational fluids in previous work by the author. ${ }^{11}$ 


\section{A CONTINUUM OF REPRESENTATIONS}

From either the Characteristic condition or Eq. (7) of section 2.2 one can see that the geometry of the bicharacteristic curves is immune to scaling by an arbitrary positive definite function, denoted by $\Omega^{2}\left(x^{\mu}\right)$. This type of invariance is called conformal symmetry. ${ }^{12}$ Conformal symmetry can be used to transform the ray equations into a continuum of equivalent representations. There are several popular representations in the acoustics literature such as time, arc-length, and Newton. ${ }^{22,23}$ A conformal transformation is defined as $\left(g_{\mu \nu}, d \lambda\right) \rightarrow \Omega^{2}\left(x^{\mu}\right)\left(g_{\mu \nu}, d \lambda\right)$, i.e., the metric and the affine parameter are both transformed while leaving the coordinates unchanged. Under this transform, the connection coefficients are changed $\Gamma^{\mu}{ }_{\alpha \beta} \rightarrow \Gamma^{\mu}{ }_{\alpha \beta}+C^{\mu}{ }_{\alpha \beta}$ with $C^{\mu}{ }_{\alpha \beta}=\delta_{\beta}^{\mu} \partial_{\alpha} \ln \Omega+$ $\delta_{\alpha}^{\mu} \partial_{\beta} \ln \Omega-g_{\alpha \beta} g^{\mu \nu} \partial_{\nu} \ln \Omega$. An important consequence of this symmetry is that the form of Eq. (5) is not changed as a result of a conformal transformation. All that is required is that the Christoffel symbols be replaced using the above prescription and a redefinition of the affine parameter. This type of transformation will not introduce additional terms in Eq. (5) as commonly happens when a generic parameter change is made. Conformally, equivalent descriptions of ray path geometry are mentioned in Babich and Kiselev. ${ }^{5}$ This topic will be revisited in the next section in relation to dynamic ray tracing.

\section{GEODESIC DEVIATION AND 4D DYNAMIC RAY TRACING}

The paradigm introduced in previous sections suggests that rays be treated as 4D curves in space-time rather than curves in $3 \mathrm{D}$ space with time as the independent parameter. The geodesic equation may be solved numerically as an initial value problem, i.v.p., or a two-point boundary value problem, b.v.p., using the affine parameter as the independent parameter. Geometric spread of the rays can be used to calculate the intensity along a ray path and, using the Blokhintzev invariant, estimate the field amplitude. ${ }^{20}$ The geodesic deviation vector, which measures the rate at which geodesics spread apart or squeeze together, provides a generalization to the law of geometric spread. $^{8,9}$ Points on nearby ray paths are described relative to a fixed ray using the original ray coordinates and a deviation vector, $x^{\mu}+Y^{\mu}$. The deviation vector, denoted $Y^{\mu}$, is chosen to be orthogonal to the ray path in $4 \mathrm{D}$ space time, $g_{\mu \nu} Y^{\mu} \dot{x}^{\nu}=0$ and its evolution is governed by the Riemann curvature tensor, $R_{\alpha \nu \beta}^{\mu}=\partial_{\nu} \Gamma^{\mu}{ }_{\alpha \beta}-\partial_{\beta} \Gamma^{\mu}{ }_{\alpha \nu}+\Gamma^{\mu}{ }_{\lambda \nu} \Gamma^{\lambda}{ }_{\alpha \beta}-$ $\Gamma_{\lambda \beta}^{\mu} \Gamma_{\alpha \nu}^{\lambda}$. The equation of geodesic deviation can be derived from Eq. (5) by considering a variation in coordinates $x^{\mu}+Y^{\mu}$, expanding all terms, and keeping only the lowest order terms in $Y^{\mu}$ and its derivatives. Focusing exclusively on the null geodesics of $M$ confines $Y^{\mu}$ to a space-like plane. Strictly speaking $Y^{\mu}$ can be expressed as a differential form, $Y^{\mu}=\frac{\partial x^{\mu}}{\partial \theta_{0}} d \theta_{0}+\frac{\partial x^{\mu}}{\partial \varphi_{0}} d \varphi_{0}$, where the deviation in ray coordinates is taken with respect to the initial ray parameters (and it has been assumed that the initial position is the same in this deviation). With this identification in place, the components of $Y^{\mu}$ are equivalent to the derivative of the ray path with respect to initial conditions. A ray centered basis is chosen for describing these deviation vectors consisting of $\frac{d x^{\mu}}{d \lambda}$ and two spacelike unit vectors, $\left\langle\hat{e}_{I}\right\rangle, I=1,2$, that are parallelly transported along the ray path by Eq. (16).

$$
\frac{d \hat{e}_{I}^{\alpha}}{d \lambda}+\Gamma_{\mu \nu}^{\alpha} \dot{x}^{\mu} \hat{e}_{I}^{\nu}=0
$$

In this local basis, the deviation vector complies with the following second order linear differential equation.

$$
\frac{d^{2} Y_{I}}{d \lambda^{2}}+K_{\mathrm{IJ}} Y_{J}=0
$$

In Eq. (17) $Y_{I}=\hat{e}_{I}^{\alpha} g_{\alpha \beta} Y^{\beta}$ is the projection of $Y^{\beta}$ onto the geodesic centered frame field, and the sectional curvature matrix $K_{\mathrm{IJ}}=R_{\mu \alpha \nu \beta} \dot{x}^{\alpha} \dot{x}^{\beta} \hat{e}_{I}^{\mu} \hat{e}_{J}^{\nu}$ is introduced. ${ }^{15}$ This provides a full system of equations for describing the ray path, travel time, and geometric spread at every point along a ray path.

$$
\frac{d}{d \lambda}\left[\begin{array}{c}
x^{\mu} \\
p^{\mu} \\
e_{I}^{\mu} \\
Y_{I} \\
P_{I}
\end{array}\right]=\left[\begin{array}{c}
p^{\mu} \\
-\Gamma^{\mu}{ }_{\alpha \beta} p^{\alpha p} \beta \\
-\Gamma^{\mu}{ }_{\alpha \beta} p^{\alpha e_{I}} \beta \\
P_{I} \\
-R_{\mu \alpha \nu \beta} p^{\alpha p} \beta e_{I}^{\mu e_{J}} \nu Y_{J}
\end{array}\right]
$$

Using the standard methods for writing these equations as a first order, ODE system leads to the following complete 4D ray trace procedure (letting $p^{\mu}=\frac{d x^{\mu}}{d \lambda}$ and $P_{I}=\frac{d Y_{I}}{d \lambda}$ ). In terms of one vector, $y=\left[x^{\mu}, p^{\mu}, \hat{e}_{I}^{\mu}, Y_{I}, P_{I}\right]^{T}$ with 20 degrees of freedom Eq. (18) is in standard form, $\frac{\mathrm{dy}}{d \lambda}=F(y)$. The use of the variables $p^{\mu}$ and $P_{I}$ for reduction of order in this section are not to be confused for generalized momentum in section 2 . The ray coordinates can be initialized at $\lambda=0$, by setting $x_{0}^{\mu}=\left(0, \vec{x}_{0}\right)$ and $\left.\frac{d x^{\mu}}{d \lambda}\right|_{0}=\left(1,(c \hat{n}+\vec{v})_{0}\right)$, where the subscript 0 refers to evaluating at the initial position. The basis vector, $\hat{e}_{I}^{\mu}$, is initialized with the time component equal to zero, $\hat{e}_{I}^{0}(0)=0$. The spatial components of one of the vectors, e.g., $\hat{e}_{1}^{k}(0)$, may be chosen by rotating $\hat{n}_{0}$ by $90^{\circ}$ in any Cartesian direction, and the second by taking $\hat{e}_{2}^{k}(0)=\hat{n}_{0} \times \hat{e}_{1}^{k}(0)$. The components of the deviation vector and its first derivative are then initialized $Y_{I}=0,\left.\frac{d Y_{I}}{d \lambda}\right|_{0}=c_{0}$. Initialization for other cases can be found in the references. ${ }^{8,9}$ The equations of constraint reduce the number of degrees of freedom significantly. The values of $Y_{I}$ at each step are used to calculate the intensity from the inverse of the cross-sectional area of a ray bundle via $\left|Y_{1} \times Y_{2}\right|$. The presence of caustics is predicted by searching for zeroes of $Y_{I}$, and the order of the caustic is determined by whether one or both deviation vectors vanish simultaneously. Field calculations near caustics require alternate methods such as WKB approximation to predict the field amplitude as is a standard practice. ${ }^{21}$

From a physical point of view, the affine parameter in 4D has no meaning and does not have an impact on the environment or the ray structure. The environmental parameters will never have explicit dependence on $\lambda$. Implementing a standard Runge-Kutta procedure involves discretizing Eq. (18). The dependent variables, $y_{n+1}$, are evaluated at each step as a weighted sum over several evaluations of the r.h.s. of Eq. (18), i.e. $y_{n+1}=y_{n}+h \sum_{i=1}^{s} c_{i} k_{i}, k_{i}=$ $F\left(\lambda_{n}+b_{i} h, y_{n}+\sum_{j=1}^{i-1} a_{\mathrm{ij}} k_{j}\right), h=\Delta \lambda$ is a discrete step, and $c_{i}, b_{i}, a_{\mathrm{ij}}$ are the weights, nodes, and elements of the Runge-Kutta matrix respectively. ${ }^{24}$ The coefficients $\left\{b_{k}\right\}$ arise from explicit dependence of the equation on the independent variable. The geodesic equation does not require these parameters for updating the independent parameter, i.e., $k_{i}=$ $F\left(y_{n}+\sum_{j=1}^{i-1} a_{\mathrm{ij}} k_{j}\right){ }^{24}$ 


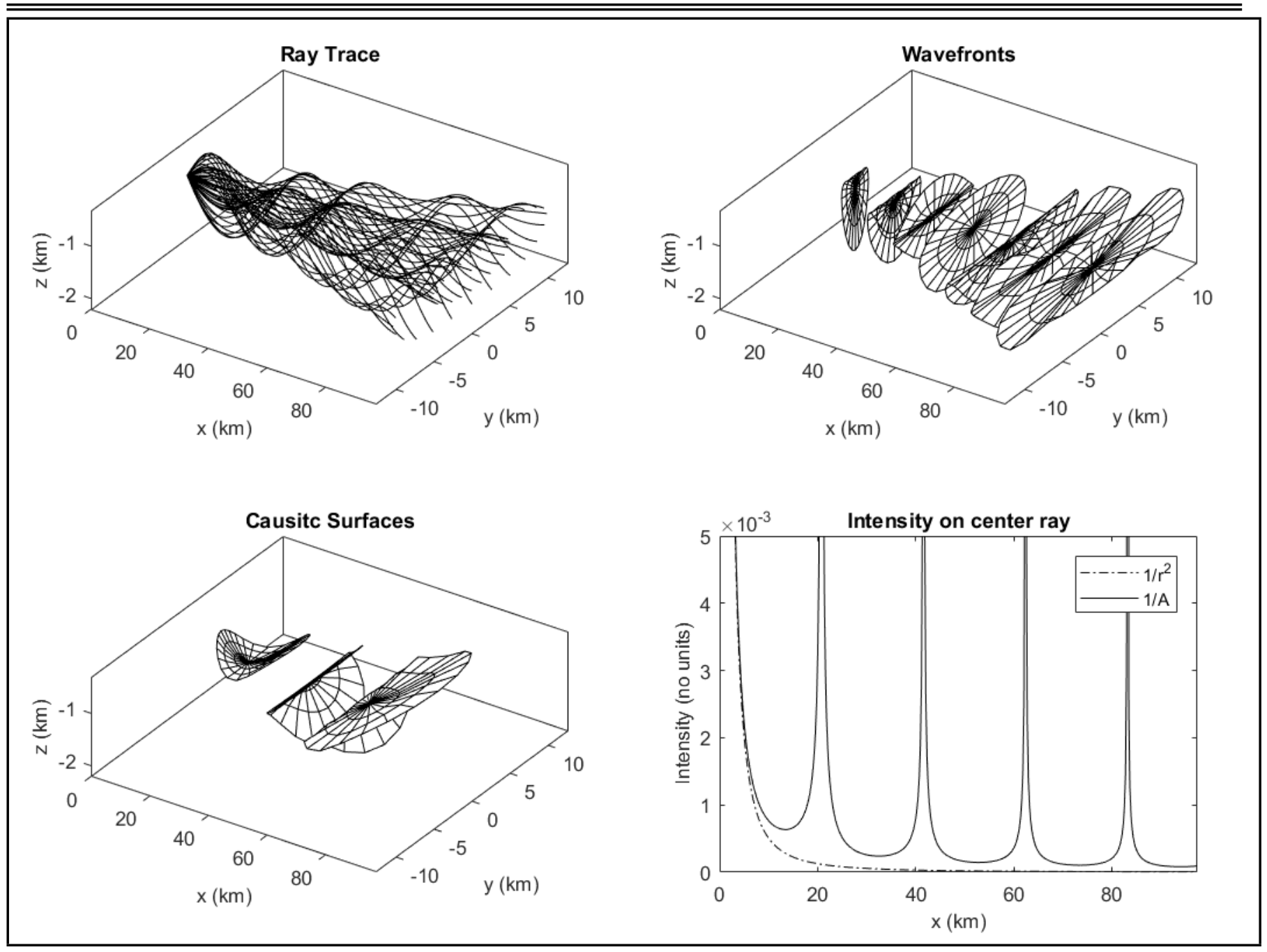

Figure 2. Sample 3D ray trace, wavefront, caustic, and intensity for the Munk sound speed.

A possible concern is the evaluation of the components of the Christoffel symbols and the Riemann curvature tensor. There is no more complexity in evaluating these quantities than evaluating the deviation of the ray equations from scratch as is often done in the literature. Ultimately there are only four quantities, $c$ and $\vec{v}$, that depend on $x^{\mu}$. Evaluating the Christoffel symbols involves taking first order derivative of these four quantities. To evaluate Riemann, one needs only take second order derivatives and form the appropriate combinations. The exact same evaluations would need to be completed in any other presentation of such a problem. It helps to view the acoustic metric, Christoffel symbol, and Riemann tensor as organizational tools for storing these quantities in such a way that condenses the final expressions for the dynamic ray equations to those found in Eqs. (5), (16), and (17). One still needs a tool for computing these quantities and this can be facilitated by commercial software packages such as Maple or Mathematica. The author is not endorsing one specific package but has experience using the tensor package found in Maple. This package will generate all associated quantities for a user supplied metric tensor. To assist the reader interested in getting started with this package a code snippet, recently executed in Maple 2016, is provided that will produce Christoffel and Riemann tensor components for the metric given in Eq. (6), where $\mathrm{c}$ is the sound speed and the three quantities vx, vy, and vz are the components of $\vec{v}$.
[> restart:

[ $>$ with(tensor):

[>cds: $=[\mathrm{t}, \mathrm{x}, \mathrm{y}, \mathrm{z}]$ :

[> g:=array (symmetric, sparse, $1 . .4,1 . .4)$ : $\mathrm{g}[1,1]:=-\left(\mathrm{c}(\mathrm{t}, \mathrm{x}, \mathrm{y}, \mathrm{z})^{\wedge} 2-\left(\mathrm{vx}(\mathrm{t}, \mathrm{x}, \mathrm{y}, \mathrm{z})^{\wedge} 2\right.\right.$ $\left.\left.+\mathrm{vy}(\mathrm{t}, \mathrm{x}, \mathrm{y}, \mathrm{z})^{\wedge} 2+\mathrm{vz}(\mathrm{t}, \mathrm{x}, \mathrm{y}, \mathrm{z})^{\wedge} 2\right)\right): \mathrm{g}[2,2]:=1:$

$\mathrm{g}[3,3]:=1: \mathrm{g}[4,4]:=1: \mathrm{g}[1,2]:=-\mathrm{vx}(\mathrm{t}, \mathrm{x}, \mathrm{y}, \mathrm{z})$ :

$\mathrm{g}[1,3]:=-\mathrm{vy}(\mathrm{t}, \mathrm{x}, \mathrm{y}, \mathrm{z}): \mathrm{g}[1,4]:=-\mathrm{vz}(\mathrm{t}, \mathrm{x}, \mathrm{y}, \mathrm{z})$ :

[> g_co:=create $([-1,-1], \operatorname{eval}(\mathrm{g}))$ :

[ $>$ tensorsGR(cds,g_co,g_ctr,g_det, C1, C2, Rm, Rc, R, G, C): [ $>$ display_allGR (cds,g_co,g_ctr, g_det, C1, C2, Rm, Rc, R, G, C);

The output quantities, defined by the user, are cds = $[t, x, y, z], g_{-}$co $=g_{\mu \nu}, g_{-} c t r=g^{\mu \nu}, \mathrm{C} 1=\Gamma_{\mu, \alpha \beta}, \mathrm{C} 2=\Gamma^{\mu \beta}{ }_{\alpha \beta}$, $\mathrm{Rm}=R_{\mu \nu \alpha \beta}, \mathrm{Rc}=$ Ricci tensor, $\mathrm{R}=$ scalar curvature, $\mathrm{G}=$ Einstein tensor, $\mathrm{C}=$ Weyl tensor. Additionally, symmetries exist that reduce the number of independent elements of these quantities, e.g. $\Gamma_{\alpha \beta}^{\mu}=\Gamma_{\beta \alpha}^{\mu}, R_{\alpha \beta \mu \nu}=-R_{\beta \alpha \mu \nu}=-R_{\alpha \beta \nu \mu}$, etc., which may be found in the references. ${ }^{18}$ Once these quantities are defined, constructing Eq. (18) is reduced to linear algebra and becomes identical for any possible environment. The heavy lifting in constructing a dynamic ray tracing system is moved to defining $g_{\alpha \beta}, \Gamma^{\mu}{ }_{\alpha \beta}$, and $R_{\alpha \beta \mu \nu}$, all of which has been automated.

Figure 2 shows sample results from the dynamic ray trace procedure for a fan of rays propagating in the presence of the Munk sound speed described by the formula, $c(z)=$ 
Table 1. Three distinct representations of the paraxial ray system.

\begin{tabular}{|l|l|l|}
\hline $\begin{array}{l}\text { Affine } \\
\text { parameter }\end{array}$ & Paraxial ray equations & $\begin{array}{l}\text { Conformal } \\
\text { transform }\end{array}$ \\
\hline$s$ & $\dot{t}=c^{-1}$ & $d s=d \lambda / c$ \\
arc-length & $\ddot{r}-\dot{r}(\dot{r} \cdot \nabla \ln c)+c^{-1} \nabla c=0$ & \\
& $\ddot{Y}+\frac{1}{2 c^{2}}\left\{\hat{u}^{i} \hat{u}^{j}+\delta^{\mathrm{ij}}\right\}\left\{c \partial_{i} \partial_{j} c-\partial_{i} c \partial_{j} c\right\} Y=0$ & \\
\hline$\lambda$ & $\dot{t}=c^{-2}$ & $d \lambda=c^{2} \mathrm{dt}$ \\
Newton & $\ddot{r}+c^{-3} \nabla c=0$ & \\
\hline $\begin{array}{l}\ddot{Y}+\left\{\hat{u}^{i} \hat{u}^{j} c^{-3} \partial_{i} \partial_{j} c\right\} Y=0 \\
\text { Time }\end{array}$ & $\ddot{r}-2 \dot{r}(\dot{r} \cdot \nabla \ln c)+c \nabla c=0$ & $d t=d s / c$ \\
\hline
\end{tabular}

Table 2. Quick reference relating differential geometry to ray theory.

\begin{tabular}{|l|l||}
\hline \multicolumn{2}{|c|}{ Relationship between geodesic flows and ray geometry } \\
\hline Geodesic & Ray \\
\hline $\begin{array}{l}\text { Geodesic } \\
\text { Polar Map }\end{array}$ & Ray skeleton and wavefronts for point source \\
\hline $\begin{array}{l}\text { Conjugate } \\
\text { Point }\end{array}$ & Caustic \\
\hline $\begin{array}{l}\text { Geodesic } \\
\text { Deviation }\end{array}$ & Cross section of ray bundle, geometric spread \\
\hline $\begin{array}{l}\text { Isometry, } \\
\text { killing vector }\end{array}$ & $\begin{array}{l}\text { Cyclic coordinate, conserved quantity Leads to } \\
\text { ray integrals in layered media }\end{array}$ \\
\hline $\begin{array}{l}\text { Conformal } \\
\text { invariance }\end{array}$ & $\begin{array}{l}\text { Ray geometry not dependent on local density } \\
\text { changes Leads to a continuum of equivalent ODE } \\
\text { systems }\end{array}$ \\
\hline
\end{tabular}

$c_{0}\left(1+\varepsilon\left(e^{-\eta}+\eta-1\right)\right)$ with $\eta=2\left(z-z_{a}\right) / B$. The specific values used to generate these plots are $c_{0}=1500 \mathrm{~m} / \mathrm{s}$, $\varepsilon=0.0057, z_{a}=1000 \mathrm{~m}, B=1000 \mathrm{~m}$.

Clockwise starting at the upper left are (1) a 3D ray trace, (2) 2D wavefronts or surfaces of constant time, (3) the first three caustic surfaces, and (4) the intensity evaluated along the central ray path due to geometric spread using the deviation vector. The initial conditions on the ray paths were chosen to generate a set of nested cones with symmetry axis pointing along the $x$-axis. Each ray is parameterized by the cone angle, $\theta_{0}$, and an azimuth, $\varphi_{0}$, about the cone axis. The cone angle increments are $0.5^{\circ}$ from $0^{\circ}$ to $8^{\circ}$. Each cone is sampled in $5^{\circ}$ from $0^{\circ}$ to $360^{\circ}$. The rays emanate from a point placed on the waveguide axis, at $z=z_{a}$. Ray traces were created using a standard ODE solver and wavefronts were generated by propagating the solver to a set of fixed specified times from $0.0 \mathrm{sec}$ to $65.0 \mathrm{sec}$ in increments of $0.15 \mathrm{sec}$ and placing a time error threshold of $5.0 \times 10^{-6} \mathrm{sec}$. Caustic surfaces were generated by searching for changes in sign of the deviation vectors and applying a linear fit to estimate the true zero crossing. Points on the wavefronts and caustic surfaces are also parameterized by $\left(\theta_{0}, \varphi_{0}\right)$. The estimated relative intensity is for the central ray only.

Conformal symmetry applies to the geodesic deviation vector as well. The internal basis vectors and deviation transform as $\hat{e}_{I}^{\mu} \rightarrow \Omega^{-1} \hat{e}_{I}^{\mu}$ and $Y_{I} \rightarrow \Omega Y_{I}$ respectively. The coordinate components of the deviation, $Y^{\mu}$, are invariant like the coordinates. The Riemann curvature tensor transforms as $R_{\alpha \nu \beta}^{\mu} \rightarrow R^{\mu}{ }_{\alpha \nu \beta}+S^{\mu}{ }_{\alpha \nu \beta}$ with the additive correction $S^{\mu}{ }_{\alpha \nu \beta}=D_{\nu} C^{\mu}{ }_{\alpha \beta}-D_{\beta} C^{\mu}{ }_{\alpha \nu}+C^{\mu}{ }_{\lambda \nu} C^{\lambda}{ }_{\alpha \beta}-C^{\mu}{ }_{\lambda \beta} C^{\lambda}{ }_{\alpha \nu}$. In this expression $D_{\nu} V^{\alpha}=\partial_{\nu} V^{\alpha}+\Gamma^{\alpha}{ }_{\nu \mu} V^{\mu}$ is the covariant derivative relative to $g_{\mu \nu}$. The transformed variables comply with Eq. (17) and the entire ray system is unchanged. As an illustrative example, the full set of paraxial ray equations is presented in Table 1. for a position dependent sound speed and no background flow, restricted to a $2 \mathrm{D}$ plane, rather than 3D. The full set of equations for three common parameterizations of the ray equation, arc-length, time, and Newton parameter (so named because the ray equations take the form of Newton's second law with a conservative force field). Isometry reduces the equation for the time variable to first order, and the equations for the single basis vector, $\hat{e}^{\mu}$, are now superfluous. Rather than track the evolution of $\hat{e}^{\mu}$, a wavefront tangent is defined everywhere by a 90-degree rotation in space of the wavefront normal, indicated by $\hat{u}$. The indices $i, j$ take values 1, 2 corresponding to 2 spatial coordinates. A full 3D version of these equations would involve greater complexity as the geodesic deviation equation would involve two degrees of freedom and angle coupling between rays in different initial planes. This section closes with a list of the concepts from differential geometry and their relationship to various quantities in ray theory presented in Table 2.

\section{ADDITIONAL TOPICS}

The previous section focused on presenting a generalized dynamic ray tracing procedure. This section introduces applications to the study of ray stability, ray chaos, and Gaussian beam tracing. The curvature tensor measures the instantaneous rate of spreading of a bundle of rays with similar initial conditions about a central ray, and is related to the Lyapunov coefficient of the system. If $K_{\mathrm{IJ}}$ vanishes, rays will spread as they would in a homogeneous medium. If the eigenvalues of $K_{\mathrm{IJ}}$ are negative, then rays will diverge at a faster rate and if the eigenvalues are positive, the deviation vector will oscillate and ray bundles will converge periodically (or semi-periodically). It is commonplace to ignore currents in underwater acoustic, at least more so than in aero acoustics, due to the mean fluid velocity being very small compared to the local speed of sound. However, studies have demonstrated that while the amplitude of the current may be small their gradients and curvatures can be large. ${ }^{13,25}$ This can be illustrated by looking at examples with $c=c(z)$, and $\vec{v}=v(z) \hat{i}$ and restricting attention to rays in the $x-z$ plane. The sectional curvature, $K_{\mathrm{IJ}}$, in Eq. (17) has a single component, $K=$ $\left(c \alpha v^{\prime \prime}(1-\alpha v)-c \alpha^{2}\left(v^{\prime}\right)^{2}-\alpha c^{\prime} v^{\prime}(1-\alpha v)+\alpha^{2} c^{2} c^{\prime \prime}\right) c^{-3} \kappa_{0}^{2}$, where the constants $\alpha$, and $\kappa_{0}$ are defined in Section 3. There are four terms with distinct focusing properties. The last term is most familiar and states that the concavity of the sound speed determines the focusing of ray bundles, the second term states that the current gradient will always cause ray divergence. The first term relates the focusing properties of the current to its concavity, similar to the last term, however it is coupled to the initial launch angle creating an anisotropy in the acoustic field. The third term couples the sound speed and current gradients to the initial launch angle of the ray. The constraints of the system impose the restriction $(1-\alpha v) \geq 0$. These effects can be further illustrated by a simple waveguide example. Consider a sound speed and current with the following second order approximations, $c \approx c_{0}+c_{0}^{\prime \prime}\left(z-z_{0}\right)^{2} / 2$ and $v \approx v_{0}+v_{0}^{\prime \prime}\left(z-z_{0}\right)^{2} / 2$, with $v_{0}>0$. For a source placed at $z=z_{0}$ and rays with initial conditions $\theta_{0}=0$, and $\pi$ the sectional curvature reduces to $K \sim c_{0}^{\prime \prime} \pm v_{0}^{\prime \prime}$. With the correct alignment of waveguide axes, local currents can 


\begin{tabular}{l}
\hline \hline Table 3. Model environments and their equivalent geometry. \\
\begin{tabular}{|l|l|l|}
\hline \multicolumn{2}{|c|}{ Model Environments and their curvature } \\
\hline $\begin{array}{l}c=c_{0} \\
v=v_{0}\end{array}$ & $K=0$ & $\begin{array}{l}\text { Geometric spread is linear } \\
\text { with respect to affine } \\
\text { parameter }\end{array}$ \\
\hline $\begin{array}{l}c=c_{0}+\xi z \\
v=0\end{array}$ & $K=0$ & $\begin{array}{l}\text { Rays experience a space of } \\
\text { constant positive curvature, } \\
\text { similar to a sphere. }\end{array}$ \\
\hline $\begin{array}{l}c=c_{0} \cosh (\xi z) \\
v=0\end{array}$ & $K=\alpha^{2} \kappa_{0}^{2} \xi^{2}$ & $\begin{array}{l}\text { Rays experience a space of } \\
\text { constant negative curvature, } \\
\text { similar to a pseudosphere }\end{array}$ \\
\hline $\begin{array}{l}c=c_{0} \\
v=v_{0}+\xi z\end{array}$ & $K=-\alpha^{2} \kappa_{0}^{2} \xi^{2} / c^{2}$ \\
\hline
\end{tabular}
\end{tabular}

turn off the effects of the inhomogeneous sound speed in one direction while doubling the effect in the other. The following results are observed, $|v / c| \sim 10^{-4},\left|v^{\prime} / c^{\prime}\right| \sim 10^{-1}$, $\left|v^{\prime \prime} / c^{\prime \prime}\right| \sim 1$, when the Munk sound speed is compared to an idealized ocean undercurrent model, Wang and Muller. ${ }^{26}$

Stationary depth waveguides with periodic range dependence have been the subject of study in underwater acoustics in the $80 \mathrm{~s}$ and $90 \mathrm{~s} .{ }^{27,28}$ The stability of rays in these and similar papers uses the parabolic equation (PE) approximation where range is treated as an independent variable while $z$ and $\tan \theta$ are treated as the coordinate and its momentum. A small angle $\mathrm{PE}$ approximation to the ray equation is derived and the behavior of $z$ and $\tan \theta$ analyzed as functions of $r$. The stability of all rays in the system can be predicted or analyzed using the sectional curvature for the sound speed model in Smith et $a l .{ }^{27}$ Equating ray systems with geodesic flows allows the machinery of DG to be adopted to the analysis of ray stability and chaos without the need to apply the parabolic assumption. For a presentation of the relationship between geometry and chaos, the reader is referred to Di Bara and Cipriani. ${ }^{29}$ Table 3. presents four toy model environments, their corresponding curvature, a description of ray stability for each, and the simple geometry corresponding to each case.

Zero curvature implies the geometry of a flat plane, a constant negative curvature is unstable, producing exponential growth in the deviation vector, and constant positive curvature is stable, producing periodic conjugate points predicted by the conjugate point theorem of DG.

A modern approach to solving the wave equation in the ray paradigm makes use of Gaussian beam tracing. ${ }^{30,31}$ Following the steps in Appendix A of Porter and Bucker, a set of curvilinear coordinates $(s, n)$ is defined in the neighborhood of a specific ray, where $s$ is arclength and $n$ a distance along the direction locally perpendicular to the ray, and $f_{n}$ denotes differentiation in the normal direction. In this approach the pressure field from a monochromatic source is described by $u(s, n)=A_{0} \sqrt{\frac{c(s)}{r q(s)}} \times \exp \left(-i \omega\left\{\tau(s)+0.5\left[\frac{p(s)}{q(s)}\right] n^{2}\right\}\right)$. The travel time along the ray path defined by $\tau(s)$, horizontal range from the source by $r$, and the complex fields $q(s)$ and $p(s)$ determined by transport equations $d q / d s=c p$ and $d p / d s=-c_{\mathrm{nn}} q / c^{2}$. Following the same steps but choosing $(\lambda, n)$ as ray coordinates leads to an equation for the Gaussian amplitude identical in form to the geodesic deviation equation. The results applied to the same calculation are presented in Table 4. for three different parameterizations, arclength $(s)$, travel time $(\tau)$, and affine parameter, $(\lambda)$. These results are derived by following the same steps as in the reference with each parameterization. ${ }^{30}$ Porter and Bucker use arclength parame-
Table 4. Gaussian Beam equations in three parameterizations.

\begin{tabular}{|l|l||}
\hline \multicolumn{2}{|c|}{ Gaussian Beam Equations in three Parameterizations } \\
\hline Ray Parameter & Beam Equations \\
\hline$s$ & $\frac{d p}{d s}+\frac{c_{n n}}{c^{2}} q=0, \frac{d q}{d s}=c p$ \\
3D Arclength & $\frac{d p}{d \tau}+\frac{c_{n n}}{c} q=0, \frac{d q}{d \tau}=c^{2} p$ \\
\hline$\tau$ & \\
Travel Time & $\frac{d^{2} q}{d \lambda^{2}}+\frac{c_{n n}}{c^{3}} q=0$ \\
\hline$\lambda$ & \\
Affine Parameter & \\
\hline
\end{tabular}

terization resulting in the equations in first row of the Table. Applying travel time and affine parameterization lead to the next two rows respectively.

Of particular interest is the fact that the affine parameterization produces the equation for geodesic deviation in the variable $q(s)$. Thus, one can reuse the equations from the dynamic ray trace for evaluating the Gaussian Beam equations in $\lambda$ parameterization. Comparing Table 4. to Table 1. of Section 5. the reader will note that the general form of the equations does not follow the same pattern. While the affine representation in Table 4. is similar in form to Eq. (17), the other parameterizations of the Gaussian beam equation do not map to an equivalent dynamic ray system. This happens because the full wave equation does not share the same conformal invariance as the geometry of the bicharacteristics. To the extent that conformal symmetry is present in the full ray theoretical description of the acoustic field but not the wave equation, it should be thought of as an approximate symmetry that can be expected to fail at higher orders.

\section{DISCUSSION}

The relationship between DG and acoustics has been discussed with attention given to the history of this approach in acoustics and its relation to other areas of physics. Meant to serve as an overview of the subject, the reader interested in a deeper tutorial is encouraged to read the references by White, Unruh, as well as O'Neill's text on DG ${ }^{15}$ and the introductory chapters of Wald's text on General Relativity. ${ }^{18}$ More detailed derivations of results presented here involving isometry, and a full derivation of the geodesic deviation equation and the dynamic ray system in Eq. (18) can be found in previous work by the author. ${ }^{9,11,12}$ Among the advantages gained by viewing ray theory in terms of geodesic flows of a Lorentzian manifold are a unified approach to all acoustics problems involving ideal Newtonian fluids with subsonic flow, this unified approach extends to dynamics ray tracing and the analysis of ray stability in the most general cases. ${ }^{32}$ The methods of DG have been used to develop paraxial ray trace procedures for time dependent moving fluids and provide insight into the use of symmetry principles for deriving results. It is hoped that these techniques will prove valuable to a wider audience over time, leading to new results and interesting research.

\section{REFERENCES}

1 Courant, R., and Hilbert, D., Methods of Mathematical Physics Volumes I and II, John Wiley \& Sons, New York, (1962)

2 Morse, P. M., and Feshbach, H., Methods of Theoretical 
Physics, McGraw-Hill Book Company, Inc., New York, (1953)

${ }^{3}$ Landau, L. D., and Lifshitz, E. M., Fluid Mechanics 2nd edition, Butterworth Heinemann, Oxford, (1987)

${ }^{4}$ White, R., Acoustic ray tracing in moving inhomogeneous fluids, Journal of the Acoustical Society of America 53 (6), 1700-1704, (1973). https://dx.doi.org/10.1121/1.1913522

5 Babich, V. M. and Kiselev, A. P., Elastic Waves High Frequency Theory, Monographs and Research Notes in Mathematics, CRC Press, 2018. https://dx.doi.org/10.1201/b21845

${ }^{6}$ Meyer, R., and Schroeter, G., The Application of Differential Geometry to Ray Acoustics in Inhomogeneous and Moving Media, ACOUSTICA, 47, 105-113, (1981)

${ }^{7}$ Unruh, W., Experimental black hole evaporation?, Physical Review Letters, 46, 1351-1353, (1981). https://dx.doi.org/10.1103/PhysRevLett.46.1351

8 Bergman, D. R., NRL.MR/7140-05-8835, Application of Differential Geometry to Acoustics: Development of a Generalized Paraxial Ray-Trace Procedure from Geodesic Deviation, (2005)

9 Bergman, D. R., Generalized space-time paraxial acoustic ray tracing, Waves in Random and Complex Media 15(4), 417-435, (2005). https://dx.doi.org/10.1080/17455030500338638

10 Bergman, D. R., Differential Geometry and Ray Theory, Proc. 22nd International Congress on Sound and Vibration (ICSV 22), 2395 - 2402, (2015)

11 Bergman, D. R., Symmetry and Snell's Law, Journal of the Acoustical Society of America 118, 1278 (2005). https://dx.doi.org/10.1121/1.1979427

12 Bergman, D. R., Internal symmetry in acoustical ray theory, Wave Motion, Vol. 43, Issue 6, 508-516, (2006). https://dx.doi.org/10.1016/j.wavemoti.2006.04.001

13 Bergman, D. R., Analysis of small currents on ray stability and caustic formation in layered moving media, Journal of the Acoustical Society of America 120, 3340 (2006). https://dx.doi.org/10.1121/1.4781336

14 Born, M., and Wolf, E., Principles of Optics, 4th Edition, Pergamon Press, Oxford, (1970)

15 O'Neill, B., Elementary Differential Geometry, Academic Press, San Diego, (1966). https://dx.doi.org/10.1016/B9781-4832-3170-9.50011-7

16 Arfken, G. B and Weber, H. J., Mathematical Methods for Physicists Fifth Edition, Academic Press, San Diego, (2001)

17 Stavroudis, O. N., The Optics of Rays, Wavefronts, and Caustics, Academic Press, New York, 1972

18 Wald, R. M., General Relativity, The University of Chicago Press, Chicago, (1984). https://dx.doi.org/10.7208/chicago/9780226870373.001.0001
${ }^{19}$ Perlick V., Ray Optics, Fermat's Principle, and Applications to General Relativity, Springer-Verlag, Berlin, (2000)

20 Blokhintzev, D., The Propagation of Sound in an Inhomogeneous and Moving Medium I, The Journal of the Acoustical Society of America, Vol. 18, No. 2, 322 - 328, (1946). https://dx.doi.org/10.1121/1.1916368

${ }^{21}$ Ludwig, D., Uniform Asymptotic Expansions at a Caustic, Communications on Pure and Applied Mathematics, Vol. XIX, 215 - 250, (1966). https://dx.doi.org/10.1002/cpa.3160190207

${ }^{22}$ Robinson, A. R. and Lee, D. editors, Oceanography and Acoustic, Prediction and Propagation Models, AIP Press, American Institute of Physics, New York, (1994)

23 Červený, V., Seismic Ray Theory, Cambridge University Press, Cambridge, (2001). https://dx.doi.org/10.1017/CBO9780511529399

${ }^{24}$ Iserles, A., A First Course in the Numerical Analysis of Differential Equations, Cambridge Texts in Applied Mathematics, Cambridge University Press, (2002)

${ }^{25}$ Colosi, J. A., Geometric sound propagation (L), Journal of the Acoustical Society of America, 119(2), 705 - 708, (2006). https://dx.doi.org/10.1121/1.2159587

26 Wang, D. and Muller, P., Journal of Physical Oceanography, 1041-1057, (2002). https://dx.doi.org/10.1175/15200485(2002)032\%3c1041:EOEUSO\%3e2.0.CO;2

${ }^{27}$ Smith, K. B., Brown, M. G. and Tappert, F. D., Acoustic ray chaos induced by mesoscale ocean structure, Journal of the Acoustical Society of America, 91(4), Pt. 1, 1950-1959, (1992). https://dx.doi.org/10.1121/1.403678

${ }^{28}$ Smith, K. B., Brown, M. G. and Tappert, F. D., Ray chaos in underwater acoustics, Journal of the Acoustical Society of America, 91(4), Pt. 1, 1939 - 1949, (1992). https://dx.doi.org/10.1121/1.403677

29 Di Bari, M., Cipriani, P., Geometry and chaos on Riemann and Finsler manifolds, Planet. Space Sci., 46 (11/12), 1543-1555, 1998. https://dx.doi.org/10.1016/S00320633(98)00059-2

${ }^{30}$ Porter, M. and Bucker, H., Gaussian beam tracing for computing ocean acoustic fields, Journal of the Acoustical Society of America 82, 1349-1359 (1987). https://dx.doi.org/10.1121/1.395269

31 Smith, S. T., On Gaussian Beams Described by Jacobi's Equation, SIAM Journal of Applied Mathematics, 74 (5), (2013). https://dx.doi.org/10.1137/130915996

${ }^{32}$ Gan, W. S., Application of Gauge Theory to Acoustic Fields - Revolutionizing and Rewriting the Whole Field of Acoustics, Proceedings of the Conference in Honor of $C$ $N$ Yang's 85th Birthday, pp. 530, World Scientific, (2008). https://dx.doi.org/10.1142/9789812794185_0065 